\title{
Article \\ COVID-19 Pandemic: Huge Stress Test for Health System Could Be a Great Opportunity to Update the Workflow in a Modern Surgical Pathology
}

\author{
Antonino Belfiore $\left.{ }^{1,+} \oplus^{(}\right)$, Giovanni Centonze $\left.{ }^{2,+}{ }^{(}\right)$, Patrick Maisonneuve ${ }^{3}\left(\mathbb{D}\right.$, Carla Riva $^{2}$, Daniele Morelli ${ }^{4}$, \\ Alessandro Mangogna ${ }^{5}$, , Giovanna Sabella ${ }^{2}$, Giancarlo Pruneri ${ }^{1, *}$ and Massimo Milione ${ }^{2, *}$
}

1 Second Pathology Unit, Department of Pathology and Laboratory Medicine, Fondazione IRCCS Istituto Nazionale dei Tumori Milano, 20133 Milan, Italy; antonino.belfiore@istitutotumori.mi.it

2 First Pathology Unit, Department of Pathology and Laboratory Medicine, Fondazione IRCCS Istituto Nazionale dei Tumori Milano, 20133 Milan, Italy; giovanni.centonze@istitutotumori.mi.it (G.C.); carla.riva@istitutotumori.mi.it (C.R.); giovanna.sabella@istitutotumori.mi.it (G.S.)

3 Division of Epidemiology and Biostatistics, European Institute of Oncology IRCCS, IEO, 20141 Milan, Italy; patrick.maisonneuve@ieo.it

4 Unit of Laboratory Medicine, Department of Pathology and Laboratory Medicine, Fondazione IRCCS Istituto Nazionale dei Tumori Milano, 20133 Milan, Italy; daniele.morelli@istitutotumori.mi.it

5 Institute for Maternal and Child Health, IRCCS Burlo Garofalo, 34137 Trieste, Italy; alessandro.mangogna@burlo.trieste.it

Citation: Belfiore, A.; Centonze, G.; Maisonneuve, P.; Riva, C.; Morelli, D. Mangogna, A.; Sabella, G.; Pruneri, G.; Milione, M. COVID-19 Pandemic: Huge Stress Test for Health System Could Be a Great Opportunity to Update the Workflow in a Modern Surgical Pathology. Cancers 2021, 13, 3283. https://doi.org/10.3390/ cancers13133283

Academic Editors: Gabriella D'Orazi and Mara Cirone

Received: 27 May 2021

Accepted: 25 June 2021

Published: 30 June 2021

Publisher's Note: MDPI stays neutral with regard to jurisdictional claims in published maps and institutional affiliations.

Copyright: (C) 2021 by the authors Licensee MDPI, Basel, Switzerland. This article is an open access article distributed under the terms and conditions of the Creative Commons Attribution (CC BY) license (https:// creativecommons.org/licenses/by/ $4.0 /)$.
* Correspondence: giancarlo.pruneri@istitutotumori.mi.it (G.P.); massimo.milione@istitutotumori.mi.it (M.M.); Tel.: +39-0223903460 (M.M.)

+ A.B. and G.C. contributed equally to this article.

Simple Summary: The COVID-19 pandemic has hit Northern Italy's regions hard in terms of deaths since February 2020. Containment measures have been applied to avoid contagion and reduce the patient infection rate. In this manuscript, we report the experience of the Pathology Department of the Fondazione IRCCS Istituto Nazionale Tumori in Milan, during the period of the first lockdown that occurred in Lombardy from March to May 2020, focusing on the variation in terms of exams between the pre-COVID-19 and COVID-19 periods and describing the measures applied to guarantee the safeguarding of workers. Moreover, we calculated if changes introduced within the workflow affected the average diagnosis time using Turn-Around-Time (TAT) metrics released by the Lombardy Region. We showed a sharp slowdown in exams during the first wave of COVID-19 and that the measures applied for the safeguarding of the personnel turned out to be feasible and did not affect the overall performance of the Pathology Department.

Abstract: Background: On December 2019, an outbreak of atypical pneumonia, known as COVID19, was identified in Wuhan, China. This disease, characterized by the rapid human-to-human transmission of the severe acute respiratory syndrome coronavirus 2 (SARS-CoV-2), has spread rapidly in more than 200 countries. Northern Italy's regions have been hit hard in terms of deaths. Here, we report the experience of the Pathology Department of the Fondazione IRCCS Istituto Nazionale Tumori (INT) in Milan, the first Italian public cancer center, in the period of the lockdown that took place in Lombardy from March to May 2020. Method: The variation in terms of exams was calculated in two different timeframes: December 2019-February 2020 (pre-COVID-19) and March-May 2020 (COVID-19). During these periods, Turn-Around-Time (TAT) metrics released by the Lombardy Region were calculated to assess if changes applied to guarantee the safeguarding of workers affected the average diagnosis time. Results: In the COVID-19 period, there was a decrease for all the performed exams. The most considerable decrease was observed for PAP tests $(-81.6 \%)$, followed by biopsies $(-48.8 \%)$, second opinions $(-41.7 \%)$, and surgical $(-31.5 \%)$, molecular $(-29.4 \%)$ and cytological $(-18.1 \%)$ tests. Measures applied within the Pathology Department, such as digital pathology, remote working, rotations and changes in operating procedures, improved the diagnostic performance as required by the guidelines of the Lombardy Region in terms of TAT. At the same time, the measures applied for the safeguarding of the personnel turned out to be feasible and did 
not affect the overall performance of the Pathology Department. Conclusions: The sharp slowdown in cancer screening during the first wave of COVID-19 could seriously endanger cancer prevention in the near future.

Keywords: SARS-CoV-2; preventive measures; cancer screening; health management

\section{Introduction}

With over 165 million cases reported and 2 million new cases per day [1], the severe acute respiratory syndrome coronavirus 2 (SARS-CoV-2) pandemic [2,3] has hit Italy particularly hard since March 2020 [4], yielding more than 125,000 deaths in three separate waves, the third of which is currently raging. Since the beginning of the SARS-CoV2 outbreak, intensive care hospitalization in Lombardy has been very high, urging a profound reorganization of healthcare facilities through the creation of specific COVID-19 hospitals able to guarantee patient isolation and intensive care support, as well as the centralization of treatment for other life-threatening diseases such as cancer in COVID19-low specialized hubs. In this scenario, Fondazione IRCCS Istituto Nazionale Tumori (INT) in Milan, an Institute of Scientific Hospitalization and Care (IRCCS), was addressed as a hub hospital by the Lombardy Region. INT is the first Italian public cancer center and is one of the main national and international referral centers for cancer treatment. It has 482 beds and is located in a metropolitan area that includes 133 different municipalities and serves more than 3 million people. Considering all these features, INT is in a privileged position to conduct patient care, and preclinical and translational research in oncology. The Department of Pathology has 82 members and is provided with fully equipped labs for routine histopathological assessment and molecular testing. The approach to cancer diagnosis has recently been revolutionized by the introduction of the mutational paradigm, which leverages the comprehensive molecular characterization of tumors for personalized care. Accordingly, we recently applied an innovative organizational model to the INT Department of Pathology, by creating two independent and highly integrated units of surgical and molecular pathology. This structure allowed us to efficiently diagnose and comprehensively profile the case-mix of roughly 50,000 exams per year, either internal or referred by community hospitals, which traditionally represent the core business of our Institution. In this manuscript, we describe the changes in the number of exams and in the workflow of the department units after the first peak of the COVID-19 epidemic in Lombardy, also focusing on the management of biological tissues and procedures to preserve the health of workers.

\section{Materials and Methods}

\subsection{Study Design}

The main objective of this study was to quantify the negative impact of the first SARSCoV-2 outbreak on cancer patients of our Institution by focusing on the number of exams performed by the INT Department of Pathology. For this reason, we evaluated the number of exams in two different timeframes, i.e., December 2019-February 2020 (pre-COVID-19 period) and March-May 2020 (COVID-19 period). The exams were grouped into different classes-"surgical", "biopsy", "second opinion", "molecular", "cytology" and "PAP test"and differentiated by the origins of the exams, namely, (i) day hospital, (ii) inpatients and (iii) outpatients.

During the lockdown period, several to-hospital and within-hospital filters were applied to protect our Institution, as described by Valenza et al. [5]. Accordingly, procedures concerning fresh material handling (fresh sample reduction and processing) and the access of external personnel were modified in both the units of the Department of Pathology, in order to preserve the health of workers [6]. As a secondary aim, we measured the performance of the Pathology Department in diagnosis production, taking advantage of 
the Turn-Around-Time (TAT) metrics released by the Lombardy Region for the pathology laboratories accredited with the national health system: this procedure establishes that at least $90 \%$ of the diagnoses must be carried out within a timeframe pre-specified for each category of exams. To this end, the internal Pathox workflow management (Tesi Elettronica e Sistemi Informativi, Milan, Italy) software was retrospectively interrogated for TAT by comparing both the pre-COVID-19 period and COVID-19 period. Finally, the changes in the operating procedures, applied for the safeguarding of workers, were described as a model for the other Pathology Laboratories.

\subsection{Operating Procedures and Reorganization of the Working Environment}

The main modifications to the operating procedures and working environment are listed below. Table 1 summarizes the changes to each procedure with respect to the preCOVID situation.

Table 1. Summary of the pre- and post-COVID-19 operating procedures modified within the Department of Pathology.

\begin{tabular}{|c|c|c|c|}
\hline Area Involved & Pre-COVID-19 Period & COVID-19 Period & $\begin{array}{l}\text { Maintenance Post } \\
\text { COVID-19 Period }\end{array}$ \\
\hline Department access & INT personnel admitted & Authorized operators only & Yes \\
\hline \multirow{3}{*}{ Personnel } & \multirow{3}{*}{$100 \%$ onsite } & $50 \%$ clinicians onsite & \multirow{3}{*}{ To be considered } \\
\hline & & $50 \%$ biologists onsite & \\
\hline & & $85 \%$ technicians onsite & \\
\hline \multirow{3}{*}{ Gross Reduction Lab } & Standard PPE for operators & Advanced PPE for operators & To be considered \\
\hline & Fresh sampling & 24-72 h fixation before sampling & No \\
\hline & $\begin{array}{c}\text { Daily intra-operative instrument } \\
\text { decontamination }\end{array}$ & $\begin{array}{c}\text { Decontamination after every } \\
\text { intra-operative exam }\end{array}$ & Yes \\
\hline \multirow{2}{*}{ Cytology Lab } & Standard PPE for operators & Advanced PPE for operators & To be considered \\
\hline & Instruments on bench & Instruments under class I biocabinet & Yes \\
\hline \multirow[b]{2}{*}{ Flow Cytometry Lab } & Standard PPE for operators & Advanced PPE for operators & To be considered \\
\hline & Daily instrument decontamination & $\begin{array}{l}\text { Instrument decontamination between } \\
\text { samples }\end{array}$ & Yes \\
\hline \multirow[b]{2}{*}{ Cytogenetics Lab } & Standard PPE for operators & Advanced PPE for operators & To be considered \\
\hline & $\begin{array}{l}\text { Sample processed under laminal } \\
\text { hood }\end{array}$ & Sample processed under laminal hood & Yes \\
\hline \multirow[b]{2}{*}{ Molecular Lab } & Standard PPE for operators & Advanced PPE for operators & To be considered \\
\hline & $\begin{array}{c}\text { Automatization of nucleic acid } \\
\text { extraction }\end{array}$ & $\begin{array}{l}\text { Automatization of nucleic acid extraction } \\
\text { under a laminar hood }\end{array}$ & To be considered \\
\hline Digital Pathology & For research use only & For diagnostic use & To be considered \\
\hline
\end{tabular}

\section{(a) Gross examination}

All the samples were already treated in a safe environment using personal protective equipment. Before the SARS-CoV-2 outbreak, sampling procedures were performed on fresh surgery tissues. The first measure adopted was to submit all the specimens to a 24-72 h fixation with $10 \%$ buffered formalin. All the fresh surgical materials referred to our department were properly handled by a pathologist, wearing a FFP2 safety mask and single-use sterile gown under a chemical fume cabinet, to improve formalin penetration, and then fixed for $24 \mathrm{~h}$ before routine sampling. All the intraoperative examinations were performed wearing FFP2 safe masks and single-use sterile gowns. The cryostat was decontaminated after every procedure using a $95 \%$ alcoholic solution. 
(b) Cytology

All the instruments dedicated to cytology were secured under a class I biosafety cabinet. Fresh samples, such as bronchoalveolar washes, urine and expectorates were processed by operators wearing FFP2 safe masks and disposable gowns. Fluids (expectorates, Bronchoalveolar lavages and bronchial aspirates) posing a risk of aerosol generation were processed under a laminar flow hood using additional disposables such as FFP2/FFP3 masks. Steps outside the flow hood were always carried out wearing an FFP2/FFP3 mask, glasses or visors, using sealed tubes. As during the pre-COVID-19 period, alcoholic or different fixatives were avoided in order to preserve the integrity of samples and prevent coarctation or flocculation.

(c) Flow cytometry

Sample processing and immunofluorescence labeling were carried out under a laminar flow hood using individual disposables. For the steps performed outside the hood, such as centrifugation, tubes were hermetically sealed. All the instruments were properly sanitized (pipettes and hoods with bleach and common areas with hydroalcoholic solution).

(d) Cytogenetics

Cell cultures from peripheral blood and bone marrow, cell cultures for the analysis of B lymphocyte aspirates, and chromosomal preparations from blood and/or bone marrow aspirates were handled under a sterile laminar flow hood. The operators always wore double gloves, protective glasses or visors and appropriate clothing with additional disposable gowns. Surfaces and centrifuges were sanitized using hydroalcoholic solution.

\section{(e) DNA extraction}

DNA extraction from blood samples was always performed using an automated Maxwell extractor (Promega, Milan, Italy). Cartridges were loaded under a laminar flow hood with closed glass. According to the regulations published by the Center for Disease Control and Prevention CDC [7], the operators wore individual protection disposables (surgical masks, a double pair of gloves, protective glasses or visors and a gown). The instruments were sanitized according to the operating instructions. The Maxwell extractor was sanitized internally with UV rays.

\section{(f) Digital Pathology}

Slides were digitalized for diagnostic use. Images were acquired on an Aperio ScanscopeXT ${ }^{\circledR}$ (Leica Biosystems Aperio, Wetzlar, Germany) at $40 \times$ and $400 \times$ magnifications and loaded on a Network Attached Storage (NAS) server in order for them to be accessible and viewable by remotely working pathologists. Software for digital slide viewing and analysis (ImageScope, Aperio, Leica Biosystem) was installed on the pathologist's personal computer, as well as them being provided with access to the institutional virtual private network (VPN) to work remotely.

\subsection{Safety of Working Environment}

In order to prevent the possible transmission of COVID-19 within the Laboratory Department, several security measures were undertaken. On 8 March 2020, a presidential decree (Law no. 34/2020) established that up to $50 \%$ of the staff personnel could be placed in remote working. The decree was then implemented during the second peak of SARS-CoV-2 infections in October 2020, in order to further balance the need to face the pandemic and the continuity of services through flexibility in working hours. We prioritized remote working for the personnel who were actually able to work from home. In particular, pathologists who shared offices with other colleagues were equipped with software for digital slide viewing and VPN access. Accordingly, biologists were also connected to the VPN in order to enable them to use the lab software remotely. These procedures allowed obtaining a $50 \%$ reduction in onsite pathologists and biologists, respectively. By contrast, only $15 \%$ of the laboratory technician staff were able to carry out remote working, due to the need to 
manually perform working procedures on site. Rotations were established to guarantee a safe alternation between onsite and remote working. The access of personnel external to the pathology department was restricted to operators carrying samples from surgical rooms and the day hospital. All the offices of the department were already equipped with sensors unlocked through electronic badges, a measure further helping to reduce contacts among inter- and intra-department operators. The reception of patients for pathology report collection or consultation sample delivery was restricted to offices equipped by glass separators, and the opening time was reduced.

\subsection{Statistical Methods}

All the statistical analyses were performed using the $\mathrm{R}$ environment for statistical computing and graphics (R Foundation, Vienna, Austria, Version 3.6.2). Comparisons of exams among groups were performed by Fisher's exact test or the Chi square test for categorical variables. All the tests were two-sided, and $p$-values $<0.05$ were considered statistically significant.

\section{Results}

\subsection{Exam Volume}

The number and distribution of the exams carried out in the department in the preCOVID-19 and COVID-19 periods are outlined in Figures 1 and 2 and Table 2. Overall, we performed 17,178 exams, distributed as follows: 4476 (26.1\%) surgical, 3163 (18.4\%) biopsies, 1205 (7.0\%) second opinions, 3561 (20.7\%) molecular tests, 1004 (5.8\%) cytological tests and 3769 (21.9\%) PAP tests (Figure 1). Upon the COVID-19 outbreak, there was a decrease for all the performed exams (Figure 2 and Table 2). The most important decrease was observed for PAP tests ( -2599 exams, corresponding to a reduction of $81.6 \%$ ), followed by biopsies ( -1021 exams or $-48.8 \%)$, surgical exams $(-838$ or $-31.5 \%)$, molecular tests $(-613$ or $-29.4 \%)$, second opinion exams $(-317$ or $-41.7 \%)$ and cytological exams $(-100$ or $-18.1 \%$ ) (Figure 2, Table 2).

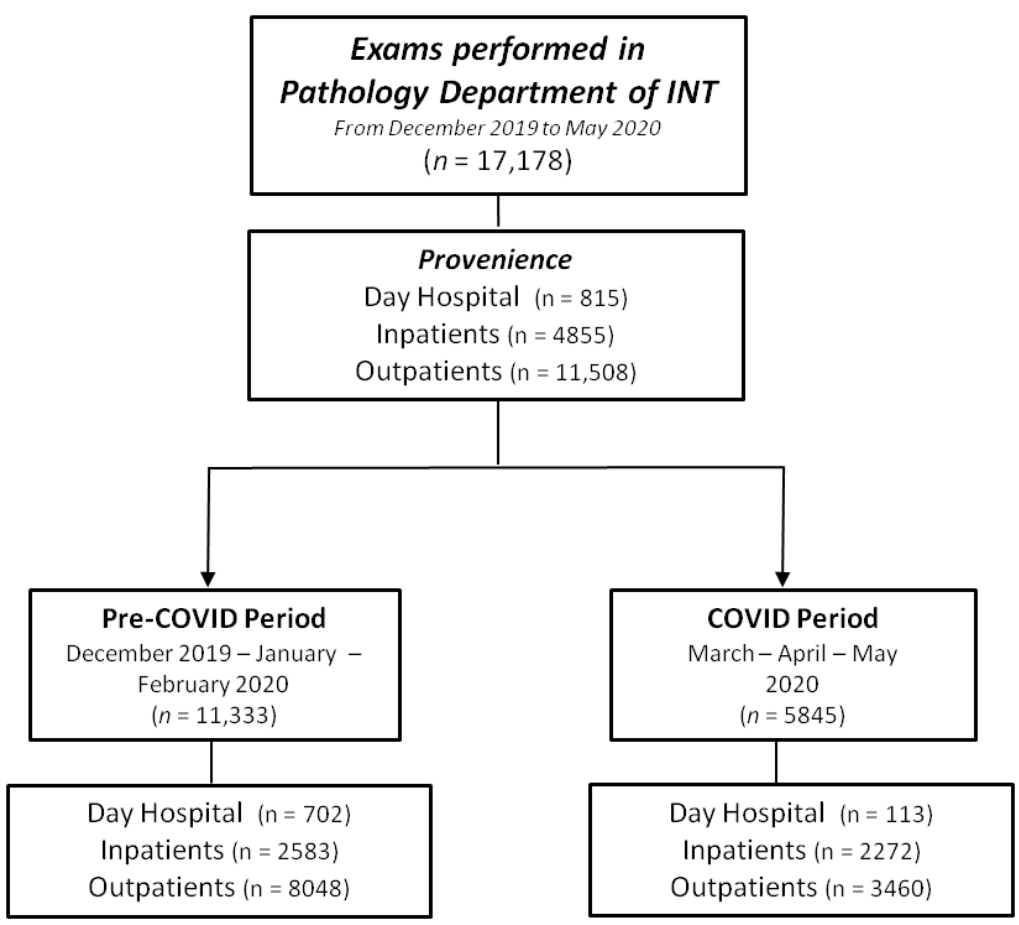

Figure 1. Flowchart of exams performed in pathology department. Abbreviations: INT, Fondazione IRCCS Istituto Nazionale Tumori of Milan (INT). 


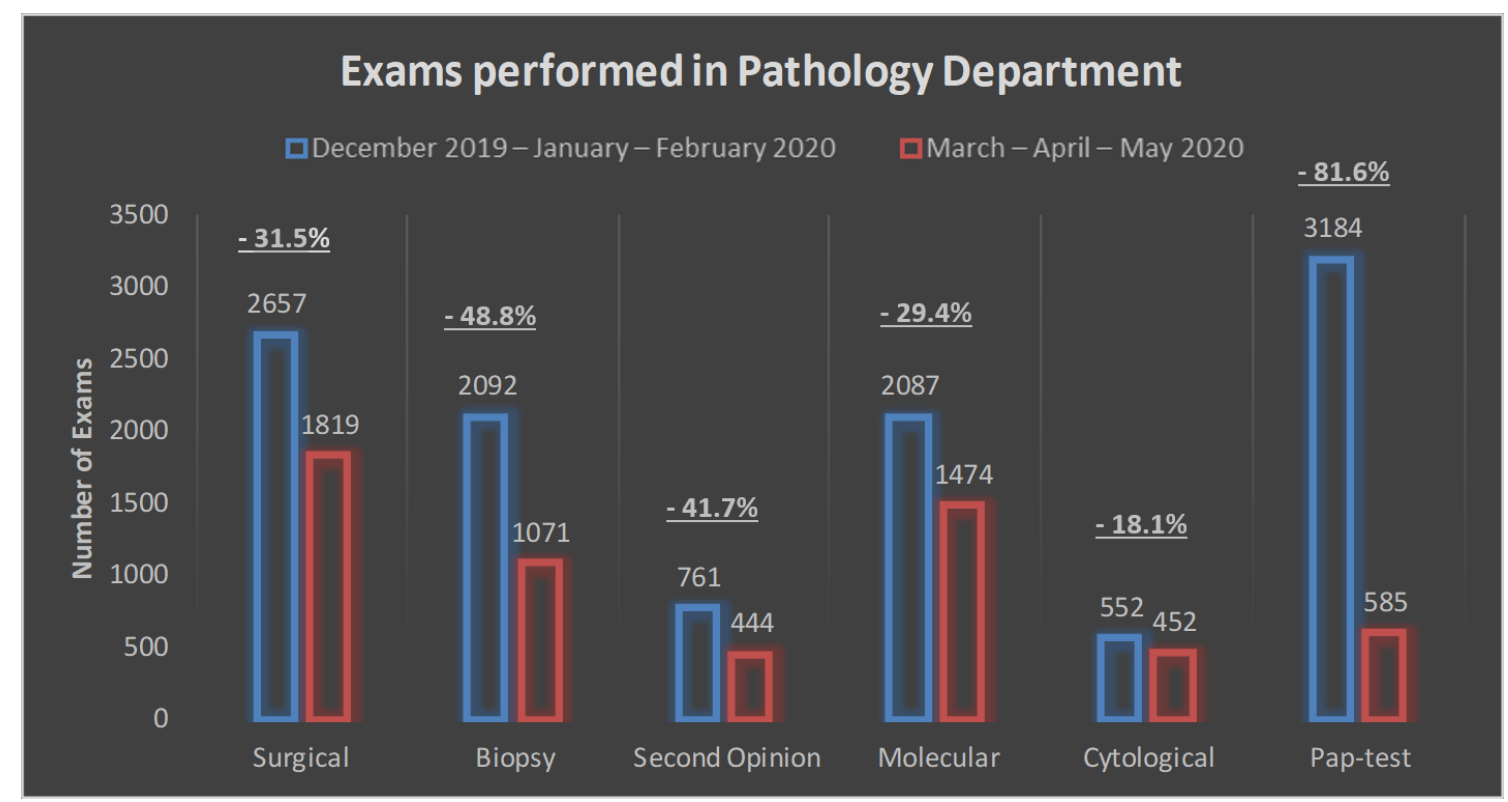

Figure 2. Number of exams performed between the pre-COVID-19 and the COVID-19 period.

Table 2. Exams performed in pre-COVID-19 and in COVID-19 quarters.

\begin{tabular}{|c|c|c|c|}
\hline Exams & $\begin{array}{c}\text { Pre-COVID-19 Period } \\
\text { (Dec 2019-Feb 2020) } \\
N(\%)\end{array}$ & $\begin{array}{c}\text { COVID-19 Period } \\
\text { (Mar-Apr 2020) } \\
N(\%)\end{array}$ & $\begin{array}{l}\text { Absolute Change } \\
\text { (Relative Change) }\end{array}$ \\
\hline All exams & $11,333(100)$ & $5845(100)$ & $-5488(-48.4 \%)$ \\
\hline All except pap test & 8149 (71.9) & $5260(90.0)$ & $-2889(-35.5 \%)$ \\
\hline Inpatients & $2583(31.7)$ & $2272(43.2)$ & $-311(-12.0 \%)$ \\
\hline Day hospital & $702(8.6)$ & $113(2.1)$ & $-589(-83.9 \%)$ \\
\hline Outpatients & $4864(59.7)$ & $2875(54.7)$ & $-1989(-40.9 \%)$ \\
\hline Surgical & $2657(23.4)$ & $1819(31.1)$ & $-838(-31.5 \%)$ \\
\hline Inpatients & $1214(45.7)$ & $1169(64.3)$ & $-45(-3.7 \%)$ \\
\hline Day hospital & $511(19.2)$ & $63(3.5)$ & $-448(-88.7 \%)$ \\
\hline Outpatients & $932(35.1)$ & $587(32.3)$ & $-345(-63.0 \%)$ \\
\hline Biopsy & $2092(18.5)$ & $1071(18.3)$ & $-1021(-48.8 \%)$ \\
\hline Inpatients & $268(12.8)$ & $188(17.6)$ & $-80(-29.9 \%)$ \\
\hline Day hospital & $143(6.8)$ & $18(1.7)$ & $-125(-87.4 \%)$ \\
\hline Outpatients & $1681(80.4)$ & $865(80.8)$ & $-816(-51.5 \%)$ \\
\hline Second opinion & $761(6.7)$ & $444(7.6)$ & $-317(-41.7 \%)$ \\
\hline Inpatients & $289(38.0)$ & $198(44.6)$ & $-91(-31.5 \%)$ \\
\hline Day hospital & $2(0.3)$ & $0(0.0)$ & $-2(-100 \%)$ \\
\hline Outpatients & $470(61.7)$ & $246(55.4)$ & $-224(-47.7 \%)$ \\
\hline Molecular & $2087(18.4)$ & $1474(25.2)$ & $-613(-29.4 \%)$ \\
\hline Inpatients & $543(26.0)$ & $443(30.0)$ & $-100(-18.4 \%)$ \\
\hline Day hospital & $20(1.0)$ & $16(1.1)$ & $-4(-20.0 \%)$ \\
\hline Outpatients & $1524(73.0)$ & 1015 (68.9) & $-509(-33.4 \%)$ \\
\hline Cytological & $552(4.9)$ & $452(7.7)$ & $-100(-18.1 \%)$ \\
\hline Inpatients & $269(48.7)$ & $274(60.6)$ & $+5(+1.9 \%)$ \\
\hline Day hospital & $26(4.7)$ & $16(3.5)$ & $-10(-38.5 \%)$ \\
\hline Outpatients & $257(46.6)$ & $162(35.9)$ & $-95(-37.0 \%)$ \\
\hline Pap test & $3184(28.1)$ & $585(10.1)$ & $-2599(-81.6 \%)$ \\
\hline
\end{tabular}




\subsection{Exam Source}

During the COVID-19 period, the distribution of the exam sources was modified. Overall, the most important reduction was observed for outpatient exams $(-1989$ or $-40.9 \%)$, followed by day hospital exams ( -589 or $-83.9 \%)$ and inpatient exams $(-311$ or $-12.0 \%$ ). Specifically, the relative frequency of surgical samples and biopsies from the day hospital dropped (19.2\% vs. $3.5 \%$ and $6.8 \%$ vs. $1.7 \%$ in the pre-COVID-19 and COVID-19 periods, respectively). Cytological exams also decreased, though to a lesser extent $(4.7 \%$ vs. $3.5 \%)$, while molecular analyses and second opinions were unaltered $(1.0 \%$ vs. $1.1 \%$ and $0.3 \%$ vs. $0 \%$, respectively). A similar trend was observed for the outpatients: the surgical ( $35.1 \%$ vs. $32.3 \%)$, cytological $(46.6 \%$ vs. $35.9 \%)$, molecular $(73.0 \%$ vs. $68.9 \%)$ and second opinion ( $61.7 \%$ vs. $55.4 \%$ ) workloads decreased during the COVID-19 period, while biopsies were unchanged $(80.8 \%$ vs. $80.4 \%)$. Finally, we registered an increase in relative frequency in all the exams within the inpatient subgroup during the COVID-19 period.

\subsection{Turn-Around-Time}

The pre-specified TAT was matched for all the test typologies, in both the pre-COVID19 and COVID-19 quarters under evaluation (Table 3). In particular, a significant increase in TAT exams was observed for surgical $(98.2 \%$ vs. $95.9 \%, p<0.0001)$ and molecular analyses $(95.8 \%$ vs. $91.8 \%, p<0.0001)$ and second opinions $(93.5 \%$ vs. $85.4 \%, p<0.0001)$ in the COVID-19 period compared to the pre-COVID-19 period. On the other hand, no significant TAT changes were observed for biopsies $(95.9 \%$ vs. $96.2 \%, p=0.69)$, cytological exams $(97.6 \%$ vs. $98.0 \%, p=0.66)$ or pap-test exams $(100 \%$ vs. $100 \%, p=1.00)$.

Table 3. Turn Around Time of exams performed in Pathology Department.

\begin{tabular}{cccc}
\hline Exams & $\begin{array}{c}\text { Pre-COVID-19 Period } \\
\text { (Dec 2019-Feb 2020) } \\
\text { N (\%) in Time }\end{array}$ & $\begin{array}{c}\text { COVID-19 Period } \\
\text { (Mar-Apr 2020) } \\
\text { N (\%) in Time }\end{array}$ & $p$-Value * \\
\hline Surgical & $2549(95.9)$ & $1786(98.2)$ & $<0.0001$ \\
Biopsies & $2013(96.2)$ & $1027(95.9)$ & 0.69 \\
Second Opinion & $650(85.4)$ & $415(93.5)$ & $<0.0001$ \\
Molecular & $1916(91.8)$ & $1412(95.8)$ & $<0.0001$ \\
Cytological & $541(98.0)$ & $441(97.6)$ & 0.66 \\
PAP Test & $3184(100)$ & $585(100)$ & 1.00 \\
\hline
\end{tabular}

* $p$-Value based on the Fisher exact test; TAT imposed by the INT for 20 working days; Abbreviations: TAT-Turn Around Time.

\section{Discussion}

The COVID-19 pandemic keeps challenging health systems worldwide, with repeated infection peaks urging deeply modifying approaches and procedures for safeguarding patients and personnel. During the first lockdown in Lombardy, lasting from March to May 2020, several structures were converted into COVID-19-hospitals, and cancer patients were centralized to a few hubs, including INT Milan. Cancer screening programs including gynecology and mammographic exams were suspended, first and follow-up visits were often postponed, and surgery was delayed except for cases defined as urgent according to specific guidelines released by the regional department of health. In this scenario, cancer centers had the twofold goal of maintaining their proficiency within a COVID-19-low environment through careful screening for COVID-19 infections and new procedures for patient triage as well as internal reorganization [5].

Here, we describe the impact of COVID-19-related procedures (the formalin fixation of fresh tissues, remote working with digital pathology and remote reporting tools) applied upon the first peak of the SARS-CoV-2 outbreak in Lombardy on the TAT of the exams carried out at the units of surgical and molecular pathology in INT Milan. The TAT measures the proficiency of a pathology lab in providing timely diagnoses, a prerequisite for properly applying personalized treatments, especially in cancer patients. We found that the TAT of all the diagnostic procedures evaluated during the COVID-19 period was 
always $>90 \%$; specifically, $93.5-100 \%$ of the exams were completed within the prespecified timeframe. This result in spite of a $50 \%$ reduction in onsite personnel (mainly pathologists and biologists) and the implementation of time-consuming procedures including $24-72 \mathrm{~h}$ formalin fixation for all the samples admitted to the department likely stems from a generalized decrease in the overall workload due to lockdown restrictions. Actually, the volume of histological, cytological and molecular procedures showed a $14.1-36 \%$ reduction during the COVID-19 period. The reduction was particularly evident for day-hospital histological and cytological exams, while samples from inpatients subjected to surgery slightly increased, a trend attributable to social confinement with the centralization of cancer patients from general hospitals. PAP tests showed the greatest decrease $(-81.6 \%)$, further confirming that cancer screening had an abrupt slowdown during the first wave of COVID-19, a trend endangering cancer prevention in the near future. These data are in keeping with a recent report of London J.W. et al. [8], who compared January-April 2019 and 2020 patient data from a network of 20 US institutions accounting for more than 28 million patients, showing a substantial decrease in breast cancer $(-89.2 \%)$ and colorectal cancer $(-84.5 \%)$ screenings. Along this line, Maringe et al. [9] predicted a substantial increase in the number of avoidable breast, colorectal, lung and esophageal cancer deaths in England as a result of diagnostic delays due to pandemic lockdown measures in the UK.

We used digital pathology to conjugate personnel safeguarding with diagnostic performance: among the procedural changes made upon the COVID-19 crisis, telemedicine is likely to be maintained and further implemented in the future. Digital pathology allows effectively making diagnoses remotely based on cytological, histological, immunohistochemical and in situ hybridization slides. When properly integrated with the institutional software and the electronical clinical record, this model permits handling all the diagnostic steps following macroscopic examination, eventually enhancing the flexibility of the personnel work schedule.

\section{Conclusions}

Collectively, our data provide evidence that implementing measures for the safeguarding of the personnel is feasible and does not affect the overall performance of the pathology laboratory. Although the model was implemented upon the first lockdown, when a significant reduction in the overall workload was observed, its efficacy was maintained in the second wave of the infection, is likely to be maintained beyond, and may be useful for other pathology laboratories included in a hub and spoke model. Finally, confirming the principle that every crisis represents an opportunity for progress, our data demonstrate that digital pathology should be implemented for a new and more efficient diagnostic approach.

Author Contributions: Study conception and design: A.B., G.C., M.M. and G.P.; data acquisition: C.R.; analysis and interpretation of data: A.B., G.C., D.M., G.S., A.M. and M.M.; drafting of manuscript: A.B. and G.C.; critical revision of the manuscript for important intellectual content: M.M., P.M. and G.P.; statistical analysis: G.C. and P.M.; study supervision: M.M. and G.P. All authors have read and agreed to the published version of the manuscript.

Funding: This study was partially supported by the Italian Ministry of Health with Ricerca Corrente and $5 \times 1000$ funds (P.M.). This work was supported by Italian Ministry of Health (ERP-2017-23671129 "PMTR-pNET" Project to M.M.).

Institutional Review Board Statement: All patients referring to Fondazione IRCCS Istituto Nazionale Tumori in Milan gave their written consent to donating the tissue remaining after diagnostic procedures. This study is based only on the number of exams extrapolated periodically and anonymously for Lombardy Region reports according to the d.g.d of 11 February 2019 n. 1606 and the d.g.r n. XI/772.

Informed Consent Statement: The patient's consent was withdrawn because only the number of exams performed, divided by categories, was used in this study without sensitive data. 
Data Availability Statement: The data presented in this study are available on request from the corresponding author.

Acknowledgments: The authors would like to thank the entire staff of the Pathology Department of INT for their commitment and dedication during the pandemic period.

Conflicts of Interest: The authors have no relevant conflicts of interest pertaining to this article.

\section{References}

1. World Health Organization. Coronavirus Disease (COVID-19) Situation Report. Available online: https://www.who.int/ emergencies/diseases/novel-coronavirus-2019/situation-reports (accessed on 25 May 2021).

2. Zhang, J.-J.; Dong, X.; Cao, Y.-Y.; Yuan, Y.-D.; Yang, Y.-B.; Yan, Y.-Q.; Akdis, C.A.; Gao, Y.-D. Clinical characteristics of 140 patients infected with SARS-CoV-2 in Wuhan, China. Allergy 2020, 75, 1730-1741. [CrossRef] [PubMed]

3. Wang, D.; Hu, B.; Hu, C.; Zhu, F.; Liu, X.; Zhang, J.; Wang, B.; Xiang, H.; Cheng, Z.; Xiong, Y.; et al. Clinical Characteristics of 138 Hospitalized Patients with 2019 Novel Coronavirus-Infected Pneumonia in Wuhan, China. JAMA 2020, 323, 1061-1069. [CrossRef] [PubMed]

4. European Centre for Disease Prevention and Control. Available online: https://www.ecdc.europa.eu/en/cases-2019-ncov-eueea (accessed on 25 May 2021).

5. Valenza, F.; Papagni, G.; Marchianò, A.; Daidone, M.G.; DeBraud, F.; Colombo, M.P.; Frignani, A.; Galmozzi, G.; Ladisa, V.; Pruneri, G.; et al. Response of a comprehensive cancer center to the COVID-19 pandemic: The experience of the Fondazione IRCCS-Istituto Nazionale dei Tumori di Milano. Tumori 2020, 300891620923790. [CrossRef] [PubMed]

6. Chen, Y.; Chen, L.; Deng, Q.; Zhang, G.; Wu, K.; Ni, L.; Yang, Y.; Liu, B.; Wang, W.; Wei, C.; et al. The presence of SARS-CoV-2 RNA in the feces of COVID-19 patients. J. Med. Virol. 2020, 92, 833-840. [CrossRef] [PubMed]

7. Centers for Disease Control and Prevention. Available online: https://www.cdc.gov/ (accessed on 25 May 2021).

8. London, J.W.; Fazio-Eynullayeva, E.; Palchuk, M.B.; Sankey, P.; McNair, C. Effects of the COVID-19 Pandemic on Cancer-Related Patient Encounters. JCO Clin. Cancer Inform. 2020, 4, 657-665. [CrossRef]

9. Maringe, C.; Spicer, J.; Morris, M.; Purushotham, A.; Nolte, E.; Sullivan, R.; Rachet, B.; Aggarwal, A.; et al. The impact of the COVID-19 pandemic on cancer deaths due to delays in diagnosis in England, UK: A national, population-based, modelling study. Lancet Oncol. 2020, 21, 1023-1034. [CrossRef] 